\title{
DIGITAL SEARCH TREES AND CHAOS GAME REPRESENTATION*
}

\author{
Peggy CénaC $^{1}$, Brigitte Chauvin ${ }^{2}$, Stéphane Ginouillac ${ }^{2}$ \\ And Nicolas PouYanne ${ }^{2}$
}

\begin{abstract}
In this paper, we consider a possible representation of a DNA sequence in a quaternary tree, in which one can visualize repetitions of subwords (seen as suffixes of subsequences). The CGRtree turns a sequence of letters into a Digital Search Tree (DST), obtained from the suffixes of the reversed sequence. Several results are known concerning the height, the insertion depth for DST built from independent successive random sequences having the same distribution. Here the successive inserted words are strongly dependent. We give the asymptotic behaviour of the insertion depth and the length of branches for the CGR-tree obtained from the suffixes of a reversed i.i.d. or Markovian sequence. This behaviour turns out to be at first order the same one as in the case of independent words. As a by-product, asymptotic results on the length of longest runs in a Markovian sequence are obtained.
\end{abstract}

Mathematics Subject Classification. 60C05, 68R15, 92D20, 05D40.

Received May 3rd, 2006. Revised March 1st, 2007.

\section{INTRODUCTION}

In the last years, DNA has been represented by means of several methods in order to make pattern visualization easier and to detect local or global similarities (see for instance Roy et al. [28]). The Chaos Game Representation (CGR) provides both a graphical representation and a storage tool. From a sequence in a finite alphabet, CGR defines a trajectory in a bounded subset of $\mathbb{R}^{d}$ that keeps all statistical properties of the sequence. Jeffrey [17] was the first to apply this iterative method to DNA sequences. Cénac [5], Cénac et al. [6] study the CGR with an extension of word-counting based methods of analysis. In this context, sequences are made of 4 nucleotides named $\mathrm{A}$ (adenine), $\mathrm{C}$ (cytosine), $\mathrm{G}$ (guanine) and $\mathrm{T}$ (thymine).

The CGR of a sequence $U_{1} \ldots U_{n} \ldots$ of letters $U_{n}$ from a finite alphabet $\mathcal{A}$ is the sequence $\left(\mathcal{X}_{n}\right)_{n \geq 0}$ of points in an appropriate compact subset $S$ of $\mathbb{R}^{d}$ defined by

$$
\left\{\begin{array}{l}
\mathcal{X}_{0} \in S \\
\mathcal{X}_{n+1}=\theta\left(\mathcal{X}_{n}+\sigma_{U_{n+1}}\right)
\end{array}\right.
$$

\footnotetext{
Keywords and phrases. Random tree, Digital Search Tree, CGR, lengths of the paths, height, insertion depth, asymptotic growth, strong convergence.

* Partially supported by the French Agence Nationale de la Recherche, project SADA ANR-05-BLAN-0372.

${ }^{1}$ INRIA Rocquencourt and Université Paul Sabatier (Toulouse III) - INRIA Domaine de Voluceau, B.P. 105, 78153 Le Chesnay Cedex, France.

${ }^{2}$ LAMA, UMR CNRS 8100, Bâtiment Fermat, Université de Versailles - Saint-Quentin, 78035 Versailles, France.
} 
where $\theta$ is a real parameter $(0<\theta<1)$, each letter $u \in \mathcal{A}$ being assigned to a given point $\sigma_{u} \in S$. In the particular case of Jeffrey's representation, $\mathcal{A}=\{A, C, G, T\}$ is the set of nucleotides, $S=[0,1]^{2}$ is the unit square. Each letter is placed at a vertex as follows:

$$
\sigma_{A}=(0,0), \quad \sigma_{C}=(0,1), \quad \sigma_{G}=(1,1), \quad \sigma_{T}=(1,0),
$$

$\theta=\frac{1}{2}$ and the first point $\mathcal{X}_{0}$ is the center of the square. Then, iteratively, the point $\mathcal{X}_{n+1}$ is the middle of the segment between $\mathcal{X}_{n}$ and the square's vertex $\sigma_{U_{n+1}}$ :

$$
\mathcal{X}_{n+1}=\frac{\mathcal{X}_{n}+\sigma_{U_{n+1}}}{2}
$$

or, equivalently,

$$
\mathcal{X}_{n}=\sum_{k=1}^{n} \frac{\sigma_{U_{k}}}{2^{n-k+1}}+\frac{\mathcal{X}_{0}}{2^{n}} .
$$

Figure 1 represents the construction of the word ATGCGAGTGT.

With each deterministic word $w=u_{1} \ldots u_{n}$, we associate the half-opened subsquare $S w$ defined by the formula

$$
S w \stackrel{\text { def }}{=} \sum_{k=1}^{n} \frac{\sigma_{u_{k}}}{2^{n-k+1}}+\frac{1}{2^{n}}\left[0,1\left[^{2} ;\right.\right.
$$

it has center $\sum_{k=1}^{n} \sigma_{u_{k}} / 2^{n-k+1}+\mathcal{X}_{0} / 2^{n}$ and side $1 / 2^{n}$. For a given random or deterministic sequence $U_{1} \ldots U_{n} \ldots$, for any word $w$ and any $n \geq|w|$ (the notation $|w|$ stands for the number of letters in $w$ ), counting the number of points $\left(\mathcal{X}_{i}\right)_{1 \leq i \leq n}$ that belong to the subsquare $S w$ is tantamount to counting the number of occurences of $w$ as a subword of $\bar{U}_{1} \ldots U_{n}$. Indeed, all successive words from the sequence having $w$ as a suffix are represented in $S w$. See Figure 1 for an example with three-letter subwords. This provides tables of word frequencies (see Goldman [15]). One can generalize it to any subdivision of the unit square; when the number of subsquares is not a power of 4 , the table of word frequencies defines a counting of words with noninteger length (see Almeida et al. [3]).

The following property of the CGR is important: the value of any $\mathcal{X}_{n}$ contains the historical information of the whole sequence $\mathcal{X}_{1}, \ldots \mathcal{X}_{n}$. Indeed, notice first that, by construction, $\mathcal{X}_{n} \in S u$ with $U_{n}=u$; the whole sequence is now given by the inductive formula $\mathcal{X}_{n-1}=2 \mathcal{X}_{n}-\sigma_{U_{n}}$.

We define a representation of a random DNA sequence $U=\left(U_{n}\right)_{n \geq 1}$ as a random quaternary tree, the $C G R$ tree, in which one can visualize repetitions of subwords. We adopt the classical order $(A, C, G, T)$ on letters. Let $\mathcal{T}$ be the complete infinite 4 -ary tree; each node of $\mathcal{T}$ has four branches corresponding to letters $(A, C, G, T)$ that are ordered in the same way. The CGR-tree of $U$ is an increasing sequence $\mathcal{T}_{1} \subset \mathcal{T}_{2} \ldots \subset \mathcal{T}_{n} \subset \ldots$ of finite subtrees of $\mathcal{T}$, each $\mathcal{T}_{n}$ having $n$ nodes. The $\mathcal{T}_{n}$ 's are built by successively inserting the reversed prefixes

$$
W(n)=U_{n} \ldots U_{1}
$$

as follows in the complete infinite tree. The first letter $W(1)=U_{1}$ is inserted in the complete infinite tree at level 1, i.e. just under the root, at the node that corresponds to the letter $U_{1}$. Inductively, the insertion of the word $W(n)=U_{n} \ldots U_{1}$ is made as follows: try to insert it at level 1 at the node $\mathcal{N}$ that corresponds to the letter $U_{n}$. If this node $\mathcal{N}$ is vacant, insert $W(n)$ at $\mathcal{N}$; if $\mathcal{N}$ is not vacant, try to insert $W(n)$ in the subtree having $\mathcal{N}$ as a root, at the node that corresponds to the letter $U_{n-1}$, and so on. One repeats this operation until the node at level $k$ that corresponds to letter $U_{n-k+1}$ is vacant; word $W(n)$ is then inserted at that node.

We complete our construction by labelling the $n$-th inserted node with the word $W(n)$. One readily obtains this way the process of a digital search tree (DST), as stated in the following proposition.

For example, Figure 2 shows the first 16 insertions in the CGR-tree that corresponds to any sequence that begins with $G A G C A C A G T G G A A G G G$. In this figure, each node has been labelled by its order of insertion to make the example more readable. 


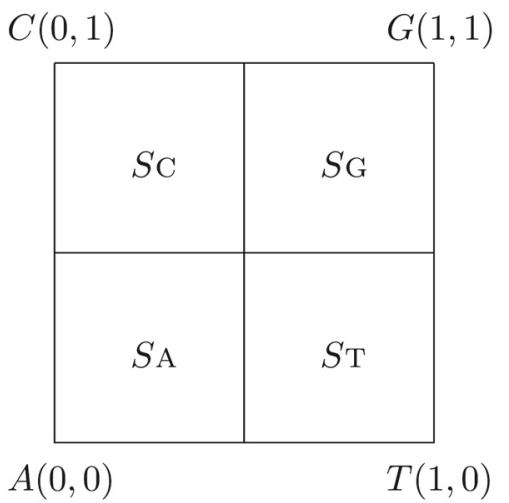

\begin{tabular}{|c|c|c|c|}
\hline \multicolumn{3}{|l|}{$C(0,1)$} & $G(1$, \\
\hline$S_{\mathrm{CC}}$ & $S \mathrm{GC}$ & $S_{\mathrm{CG}}$ & $S \mathrm{GG}$ \\
\hline$S_{\mathrm{AC}}$ & $S \mathrm{TC}$ & $S_{\mathrm{AG}}$ & $S$ TG \\
\hline$S \mathrm{CA}$ & $S \mathrm{GA}$ & $S \mathrm{CT}$ & $S \mathrm{GT}$ \\
\hline$S$ AA & $S \mathrm{TA}$ & $S$ AT & $S$ TT \\
\hline$A(0,0)$ & & & $T($ \\
\hline
\end{tabular}

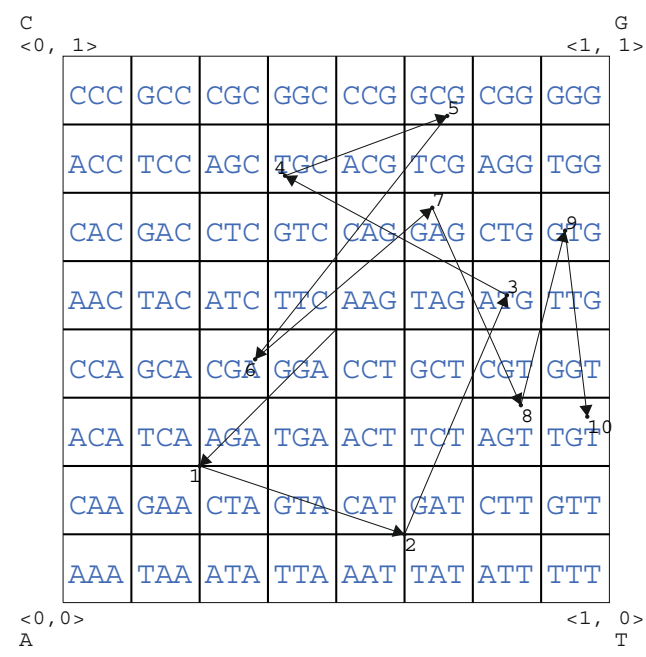

Figure 1. Chaos Game Representation of the first 10 nucleotides of the E. Coli threonine gene thrA: ATGCGAGTGT. The coordinates for each nucleotide are calculated recursively using $(0.5,0.5)$ as starting position. The sequence is read from left to right. Point number 3 corresponds to the first 3-letter word $A T G$. It is located in the corresponding quadrant. The second 3-letter word $T G C$ corresponds to point 4 and so on.

Proposition 1.1. The CGR-tree of a random sequence $U=U_{1} U_{2} \ldots$ is a digital search tree, obtained by insertion in a quaternary tree of the successive reversed prefixes $U_{1}, U_{2} U_{1}, U_{3} U_{2} U_{1}$, . of the sequence.

The main results of our paper are the following convergence results, the random sequence $U$ being supposed to be Markovian. If $\ell_{n}$ and $\mathcal{L}_{n}$ denote respectively the length of the shortest and of the longest branch of the CGR-tree, then $\ell_{n} / \ln n$ and $\mathcal{L}_{n} / \ln n$ converge almost surely to some constants (Th. 3.1). Moreover, if $D_{n}$ denotes the insertion depth of $W(n)$ in $\mathcal{T}_{n-1}$ and if $M_{n}$ is the length of a uniformly chosen random path up to a leaf, then $D_{n} / \ln n$ and $M_{n} / \ln n$ converge in probability to a common constant (Th. 4.1).

At least four tree representations of the sequence $U=U_{1} U_{2} \ldots$ are possible: its reversed prefix DST (our representation), its suffix DST, its suffix trie and its reversed prefix trie. Suffix tries have given rise to a yet incomplete literature (see Szpankowski [31] or Fayolle [11] for an overview). The prefix DST representation has been chosen here, for a better matching with the CGR representation. 

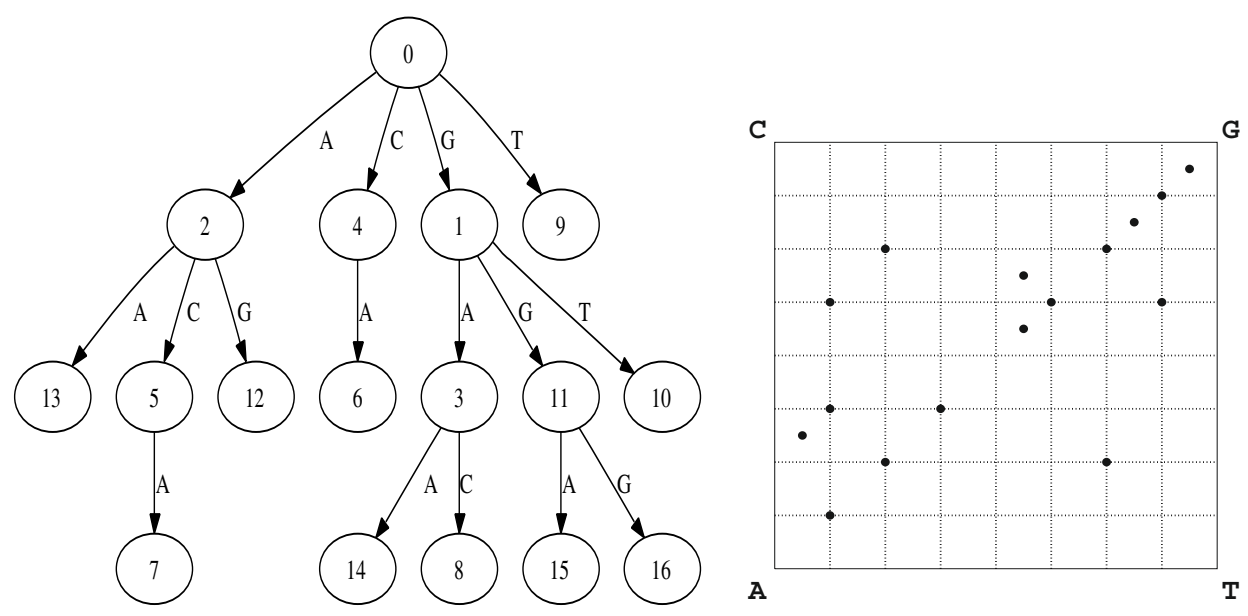

Figure 2. On the left, insertion of the 16 first keys in the CGR-tree of the DNA sequence Mus

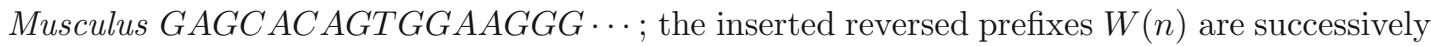
$G, A G, G A G, C G A G, A C G A G$, etc. and the nodes are labelled by the orders of insertion $n$. On the right, the corresponding "historyless representation".

Remark 1.2. A given CGR-tree without its labels (i.e. a given shape of tree) is equivalent to a list of words in the sequence without their order. More precisely, one can associate with a shape of CGR-tree, a representation in the unit square as described below. With any node of the tree (which is in bijection with a word $w=W_{1} \ldots W_{d}$ ), we associate the center of the corresponding square $S w$,

$$
\mathcal{X}_{w} \stackrel{\text { def }}{=} \sum_{k=1}^{d} \frac{\sigma_{W_{k}}}{2^{d-k+1}}+\frac{\mathcal{X}_{0}}{2^{d}} .
$$

For example, Figure 2 (right) shows this "historyless representation" for the word GAGCACAGTGGAAGGG. Moreover Figure 3 enables us to qualitatively compare the original and the historyless representations on an example.

Several results are known (see Chap. 6 in Mahmoud [20]), concerning the height, the insertion depth and the profile for DST obtained from independent successive sequences, having the same distribution. It is far from our situation where the successive inserted words are strongly dependent from each other. Various results concerning the so-called Bernoulli model (binary trees, independent sequences and the two letters have the same probability $1 / 2$ of appearance) can be found in Mahmoud [20]. Aldous and Shields [2] prove by embedding in continuous time, that the height satisfies $H_{n}-\log _{2} n \rightarrow 0$ in probability. Also Drmota [7] proves that the height of such DSTs is concentrated: $\mathbb{E}\left[H_{n}-\mathbb{E}\left(H_{n}\right)\right]^{L}$ is bounded for any $L>0$.

For DST constructed from independent sequences on an $m$-letter alphabet with nonsymmetric (i.e. non equal probabilities on the letters) i.i.d or Markovian sources, Pittel [23] gets several results on the insertion depth and on the height. Despite the independence of the sequences, Pittel's work seems to be the closest to ours, and some parts of our proofs are inspired by it.

Some proofs in the sequel use classical results on the distribution of word occurences in a random sequence of letters (independent or Markovian sequences). Blom and Thorburn [4] give the generating function of the first occurence of a word for i.i.d. sequences, based on a recurrence relation on the probabilities. This result is extended to Markovian sequences by Robin and Daudin [27]. Several studies in this domain are based on generating functions, for example Régnier [25], Reinert et al. [26], Stefanov and Pakes [30]. Nonetheless, other approaches are considered: one of the more general techniques is the Markov chain embedding method 
C

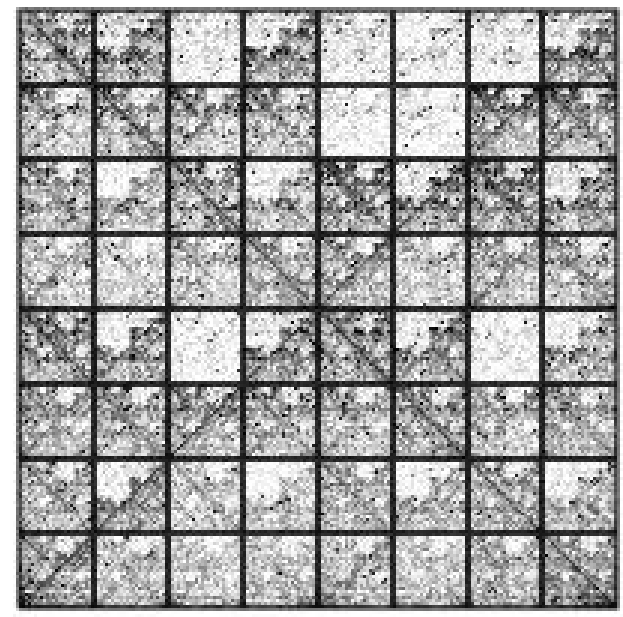

G

C

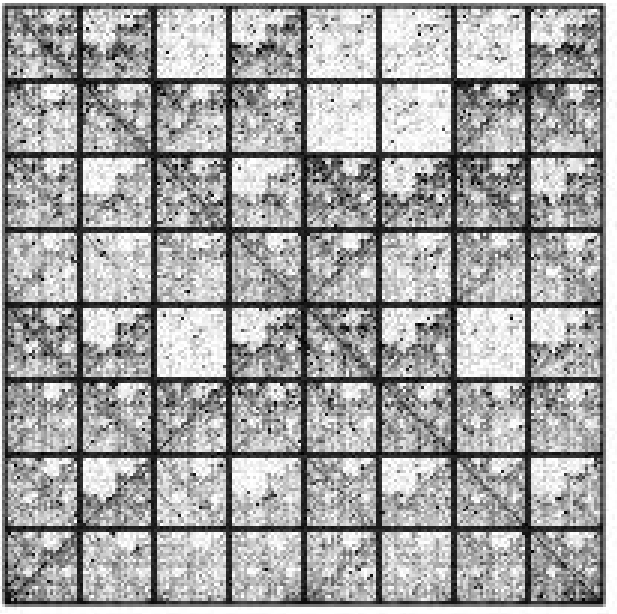

A

Figure 3. Chaos Game Representation (on the left) and historyless representation (on the right) of the first 400000 nucleotides of Chromosome 2 of Homo Sapiens.

introduced by $\mathrm{Fu}$ [12] and further developped by Fu and Koutras [13], Koutras [18]. A martingale approach (see Gerber and $\mathrm{Li}$ [14], Li [19], Williams [32]) is an alternative to the Markov chain embedding method to solve problems around Penney [21] Game. These two approaches are compared in Pozdnyakov et al. [24]. Whatever method one uses, the distribution of the first occurence of a word strongly depends on its overlapping structure. This dependence is at the core of our proofs.

As a by-product, our results provide an extension and a new proof of known asymptotic properties on the length of the longest run, which is a natural object of study (see Rem. 3.3). For i.i.d. and symmetric sequences, Erdős and Révész [9] establish almost sure results about the growth of the longest run. These results are extended to Markov chains in Samarova [29], and Gordon et al. [16] show that the probabilistic behaviour of the length of the longest run is closely approximated by that of the maximum of some i.i.d. exponential random variables.

The paper is organized as follows. In Section 2 we establish the assumptions and notations we use throughout. Section 3 is devoted to almost sure convergence of the shortest and the longest branches in CGR-trees. In Section 4 asymptotic behaviour of the insertion depth is studied. An appendix deals separately with the domain of definition of the generating function of a certain waiting time related to the overlapping structure of words.

\section{Assumptions And notations}

In all the sequel, the sequence $U=U_{1} \ldots U_{n} \ldots$ is supposed to be a Markov chain of order 1 , with transition matrix $Q$ and invariant measure $p$ as initial distribution.

For any deterministic infinite sequence $s$, let us denote by $s^{(n)}$ the word formed by the $n$ first letters of $s$, that is to say $s^{(n)} \stackrel{\text { def }}{=} s_{1} \ldots s_{n}$, where $s_{i}$ is the $i$-th letter of $s$. The measure $p$ is extended to reversed words in the following way: $p\left(s^{(n)}\right) \stackrel{\text { def }}{=} \mathbb{P}\left(U_{1}=s_{n}, \ldots, U_{n}=s_{1}\right)$. The need for reversing the word $s^{(n)}$ comes from the construction of the CGR-tree which is based on reversed sequences (1). 
We define the constants

$$
\begin{aligned}
& h_{+} \stackrel{\text { def }}{=} \lim _{n \rightarrow+\infty} \frac{1}{n} \max \left\{\ln \left(\frac{1}{p\left(s^{(n)}\right)}\right), p\left(s^{(n)}\right)>0\right\}, \\
& h_{-} \stackrel{\text { def }}{=} \lim _{n \rightarrow+\infty} \frac{1}{n} \min \left\{\ln \left(\frac{1}{p\left(s^{(n)}\right)}\right), p\left(s^{(n)}\right)>0\right\}, \\
& h \stackrel{\text { def }}{=} \lim _{n \rightarrow+\infty} \frac{1}{n} \mathbb{E}\left[\ln \left(\frac{1}{p\left(U^{(n)}\right)}\right)\right] .
\end{aligned}
$$

Using an argument of sub-additivity, it is shown in Pittel [23] that these limits are well defined (in a more general framework than that of Markovian sequences). Moreover, Pittel proves the existence of two infinite sequences denoted here by $s_{+}$and $s_{-}$such that

$$
h_{+}=\lim _{n \rightarrow \infty} \frac{1}{n} \ln \left(\frac{1}{p\left(s_{+}^{(n)}\right)}\right), \quad \text { and } \quad h_{-}=\lim _{n \rightarrow \infty} \frac{1}{n} \ln \left(\frac{1}{p\left(s_{-}^{(n)}\right)}\right) .
$$

For any $n \geq 1$, the notation $\mathcal{T}_{n} \stackrel{\text { def }}{=} \mathcal{T}_{n}(W)$ stands for the finite tree with $n$ nodes (without counting the root), built from the first $n$ sequences $W(1), \ldots, W(n)$, which are the successive reversed prefixes of the sequence $\left(U_{n}\right)_{n}$, as defined by $(1) . \mathcal{T}_{0}$ denotes the tree reduced to the root. In particular, the random trees are increasing: $\mathcal{T}_{0} \subset \mathcal{T}_{1} \ldots \subset \mathcal{T}_{n} \subset \ldots \subset \mathcal{T}$

Let us define $\ell_{n}$ (resp. $\mathcal{L}_{n}$ ) as the length of the shortest (resp. the longest) path from the root to an external node of the tree $\mathcal{T}_{n}$. Moreover, $D_{n}$ denotes the insertion depth of $W(n)$ in $\mathcal{T}_{n-1}$ to build $\mathcal{T}_{n}$. Finally $M_{n}$ is the length of a path of $\mathcal{T}_{n}$, randomly and uniformly chosen in the $n$ possible paths.

The following random variables play a key role in the proofs. For any deterministic infinite sequence $s$ and for any $n \geq 0$, we first define the random variable $X_{n}(s)$ as being the length of the branch associated with $s$ in the tree $\mathcal{T}_{n}$, i.e.

$$
X_{n}(s) \stackrel{\text { def }}{=}\left\{\begin{array}{l}
0 \text { if } s_{1} \text { is not in } \mathcal{T}_{n} \\
\max \left\{k \text { such that } s^{(k)} \text { is already inserted in } \mathcal{T}_{n}\right\} .
\end{array}\right.
$$

Observe that $X_{0}(s)=0$ and that the randomness is uniquely due to the generation of the sequence $U$, and not to the deterministic sequence $s$. Every infinite sequence corresponds to a branch of the infinite tree $\mathcal{T}$ (root at level 0 , node that corresponds to $s_{1}$ at level 1 , node that corresponds to $s_{2}$ at level 2, etc.); the random variable $X_{n}(s)$ is the length of the branch associated with $s$ in the tree $\mathcal{T}_{n}$. For any $k \geq 0$, let $T_{k}(s)$ be defined as the waiting time

$$
T_{k}(s) \stackrel{\text { def }}{=} \min \left\{n, X_{n}(s)=k\right\}
$$

(notice that $T_{0}(s)=0$ ). It is the size of the first tree where $s^{(k)}$ is inserted

These two variables are in duality in the following sense: one has equality of the events

$$
\left\{X_{n}(s) \geq k\right\}=\left\{T_{k}(s) \leq n\right\}
$$

and consequently, $\left\{T_{k}(s)=n\right\} \subset\left\{X_{n}(s)=k\right\}$ since $X_{n}(s)-X_{n-1}(s) \in\{0,1\}$.

In our example of Figure 2, the drawn random sequence is GAGCACAGTGGAAGGG... If one takes a deterministic sequence $s$ such that $s^{(3)}=A C A$, then $X_{0}(s)=X_{1}(s)=0, X_{2}(s)=X_{3}(s)=X_{4}(s)=1$, $X_{5}(s)=X_{6}(s)=2$ and $X_{k}(s)=3$ for $7 \leq k \leq 18$. The first three values of $T_{k}(s)$ are consequently $T_{1}(s)=2$, $T_{2}(s)=5, T_{3}(s)=7$.

Moreover, the random variable $T_{k}(s)$ can be decomposed as follows,

$$
T_{k}(s)=\sum_{r=1}^{k} Z_{r}(s)
$$




\begin{tabular}{|c|c|c|c|c|c|c|c|}
\hline$U_{3+T_{5}(s)}$ & $U_{2+T_{5}(s)}$ & $U_{1+T_{5}(s)}$ & $s_{1}$ & $s_{2}$ & $s_{3}$ & $s_{4}$ & $s_{5}$ \\
\hline$s_{1}$ & $s_{2}$ & $s_{3}$ & $s_{4}$ & $s_{5}$ & $s_{6}$ & & \\
\hline
\end{tabular}

FiguRE 4. How overlapping intervenes in $Z_{r}(s)^{\prime}$ definition. In this example, one takes $r=6$. In the random sequence, prefix $s^{(6)}$ can occur starting from $U_{3+T_{5}(s)}$ only if $s_{1} s_{2} s_{3}=s_{4} s_{5} s_{6}$.

where $Z_{r}(s) \stackrel{\text { def }}{=} T_{r}(s)-T_{r-1}(s)$ is the number of letters to read before the branch that corresponds to $s$ increases by 1 . In what follows, $Z_{r}(s)$ can be viewed as the waiting time $n$ of the first occurence of $s^{(r)}$ in the sequence

$$
\ldots U_{n+T_{r-1}(s)} U_{n-1+T_{r-1}(s)} \ldots U_{1+T_{r-1}(s)} s^{(r-1)}
$$

i.e. $Z_{r}(s)$ can also be defined as

$$
Z_{r}(s)=\min \left\{n \geq 1, U_{n+T_{r-1}(s)} \ldots U_{n+T_{r-1}(s)-r+1}=s_{1} \ldots s_{r}\right\} .
$$

Because the model is Markovian, the random variables $Z_{r}(s)$ are independent.

Let us then introduce $Y_{r}(s)$ as being the waiting time of the first occurence of $s^{(r)}$ in the sequence

$$
\ldots U_{n+T_{r-1}(s)} U_{n-1+T_{r-1}(s)} \ldots U_{1+T_{r-1}(s)}
$$

that is to say

$$
Y_{r}(s)=\min \left\{n \geq r, U_{n+T_{r-1}(s)} \ldots U_{n+T_{r-1}(s)-r+1}=s_{1} \ldots s_{r}\right\} .
$$

One has readily the inequality $Z_{r}(s) \leq Y_{r}(s)$. More precisely, if the word $s^{(r)}$ appears in the sequence before time $T_{r-1}(s)+r$, there is some overlapping between prefixes of $s^{(r-1)}$ and suffixes of $s^{(r)}$. See Figure 4 for an example where $r=6$ and $s_{1} s_{2} s_{3}=s_{4} s_{5} s_{6}$. Actually, variables $Z_{r}(s)$ and $Y_{r}(s)$ are related by

$$
Z_{r}(s)=\mathbb{1}_{\left\{Z_{r}(s)<r\right\}} Z_{r}(s)+\mathbb{1}_{\left\{Z_{r}(s) \geq r\right\}} Y_{r}(s) .
$$

Since the sequence $\left(U_{n}\right)_{n \geq 1}$ is stationary, the conditional distribution of $Y_{r}(s)$ given $T_{r-1}(s)$ is the distribution of the first occurence of the word $s^{(r)}$ in the realization of a Markov chain of order 1, whose transition matrix is $Q$ and whose initial distribution is its invariant measure. In particular the conditional distribution of $Y_{r}(s)$ given $T_{r-1}(s)$ is independent of $T_{r-1}(s)$.

The generating function $\Phi\left(s^{(r)}, t\right) \stackrel{\text { def }}{=} \mathbb{E}\left[t^{Y_{r}(s)}\right]$ is given by Robin and Daudin [27]:

$$
\Phi\left(s^{(r)}, t\right)=\left(\gamma_{r}(t)+(1-t) \delta_{r}\left(t^{-1}\right)\right)^{-1}
$$

where the functions $\gamma$ and $\delta$ are respectively defined as

$$
\gamma_{r}(t) \stackrel{\text { def }}{=} \frac{1-t}{t p\left(s_{r}\right)} \sum_{m \geq 1} Q^{m}\left(s_{1}, s_{r}\right) t^{m}, \quad \delta_{r}\left(t^{-1}\right) \stackrel{\text { def }}{=} \sum_{m=1}^{r} \frac{\mathbb{1}_{\left\{s_{r} \ldots s_{r-m+1}=s_{m} \ldots s_{1}\right\}}}{t^{m} p\left(s^{(m)}\right)},
$$

and where $Q^{m}(u, v)$ denotes the transition probability from $u$ to $v$ in $m$ steps.

Remark 2.1. In the particular case when the sequence of nucleotides $\left(U_{n}\right)_{n \geq 1}$ is supposed to be independent and identically distributed according to the non degenerated law $\left(p_{A}, p_{C}, p_{G}, p_{T}\right)$, the transition probability $Q^{m}\left(s_{1}, s_{r}\right)$ is equal to $p\left(s_{r}\right)$, and hence $\gamma_{r}(t)=1$. 


\section{Proposition 2.2.}

(i) The generating function of $Y_{r}(s)$ defined by (6) has a radius of convergence $\geq 1+\kappa p\left(s^{(r)}\right)$ where $\kappa$ is a positive constant independent of $r$ and $s$.

(ii) Let $\gamma$ denote the second largest eigenvalue of the transition matrix $Q$. For all $t \in]-\gamma^{-1}, \gamma^{-1}[$,

$$
\left|\gamma_{r}(t)-1\right| \leq \frac{|1-t|}{1-\gamma|t|} \kappa^{\prime},
$$

where $\kappa^{\prime}$ is some positive constant independent of $r$ and $s$ (if $\gamma=0$ or if the sequence is i.i.d., we adopt the convention $\gamma^{-1}=+\infty$ so that the result remains valid).

Proof. The proof of Proposition 2.2 is given in Appendix A.

\section{LENGTH OF THE BRANCHES}

In this section we are concerned with the asymptotic behaviour of the length $\ell_{n}$ (resp. $\mathcal{L}_{n}$ ) of the shortest (resp. longest) branch of the CGR-tree.

\section{Theorem 3.1.}

$$
\frac{\ell_{n}}{\ln n} \underset{\mathrm{n} \rightarrow \infty}{\stackrel{\text { rma.s. }}{\longrightarrow}} \frac{1}{h_{+}}, \quad \text { and } \quad \underset{\ln n}{\stackrel{\mathcal{L}_{n}}{\ln \rightarrow \infty} \underset{\text { rma.s. }}{\longrightarrow}} \frac{1}{h_{-}} .
$$

According to the definition of $X_{n}(s)$, the lengths $\ell_{n}$ and $\mathcal{L}_{n}$ are functions of $X_{n}$ :

$$
\ell_{n}=\min _{s \in \mathcal{A}^{\mathbb{N}}} X_{n}(s), \quad \text { and } \quad \mathcal{L}_{n}=\max _{s \in \mathcal{A}^{\mathbb{N}}} X_{n}(s)
$$

The following key lemma gives an asymptotic result on $X_{n}(s)$, under suitable assumptions on $s$. Our proof of Theorem 3.1 is based on it.

Lemma 3.2. Let $s$ be such that there exists

$$
\lim _{n \longrightarrow+\infty} \frac{1}{n} \ln \left(\frac{1}{p\left(s^{(n)}\right)}\right) \stackrel{\text { def }}{=} h(s)>0 .
$$

Then

$$
\frac{X_{n}(s)}{\ln n} \underset{\mathrm{n} \rightarrow \infty}{\stackrel{\text { a.s. }}{\longrightarrow}} \frac{1}{h(s)}
$$

Remark 3.3. Let $\tilde{v} \stackrel{\text { def }}{=} v v \ldots$ consist of repetitions of a letter $v$. Then $X_{n}(\tilde{v})$ is the length of the branch associated with $\tilde{v}$ in $\mathcal{T}_{n}$. For such a sequence (and exclusively for them) the random variable $Y_{k}(\tilde{v})$ is equal to $T_{k}(\tilde{v})$. Consequently $X_{n}(\tilde{v})$ is the length of the longest run of ' $v$ ' in $U_{1} \ldots U_{n}$. When $\left(U_{n}\right)_{n \geq 1}$ is a sequence of i.i.d. trials, $[9,10,22]$ showed that

$$
\frac{X_{n}(\tilde{v})}{\ln n} \underset{\mathrm{n} \rightarrow \infty}{\stackrel{\text { a.s. }}{\longrightarrow}} \frac{1}{\ln \frac{1}{p}}
$$

where $p \stackrel{\text { def }}{=} \mathbb{P}\left(U_{i}=v\right)$. This convergence result is a particular case of Lemma 3.2.

Simulations. In a first set of computations (Figs. 5 and 6), two random sequences whose letters are i.i.d. were generated.

In Figure 5, letters are drawn with respective probabilities $\left(p_{A}, p_{C}, p_{G}, p_{T}\right)=(0.4,0.3,0.2,0.1)$; in Figure 6 , letters are equally-likely drawn. One can visualize the dynamic convergence of $\mathcal{L}_{n} / \ln n, \ell_{n} / \ln n$ and of the normalized insertion depth $D_{n} / \ln n$ (see Sect. 4) to their respective constant limits.

Figure 7 is made from simulations of 2,000 random sequences of length 100,000 with i.i.d. letters under the distribution $\left(p_{A}, p_{C}, p_{G}, p_{T}\right)=(0.6,0.1,0.1,0.2)$. On the $x$-axis, respectively, lengths of the shortest branches, 
Insertion levels and shortest/longest branches

[length $=100000$, probs $=0.4,0.3,0.2,0.1]$
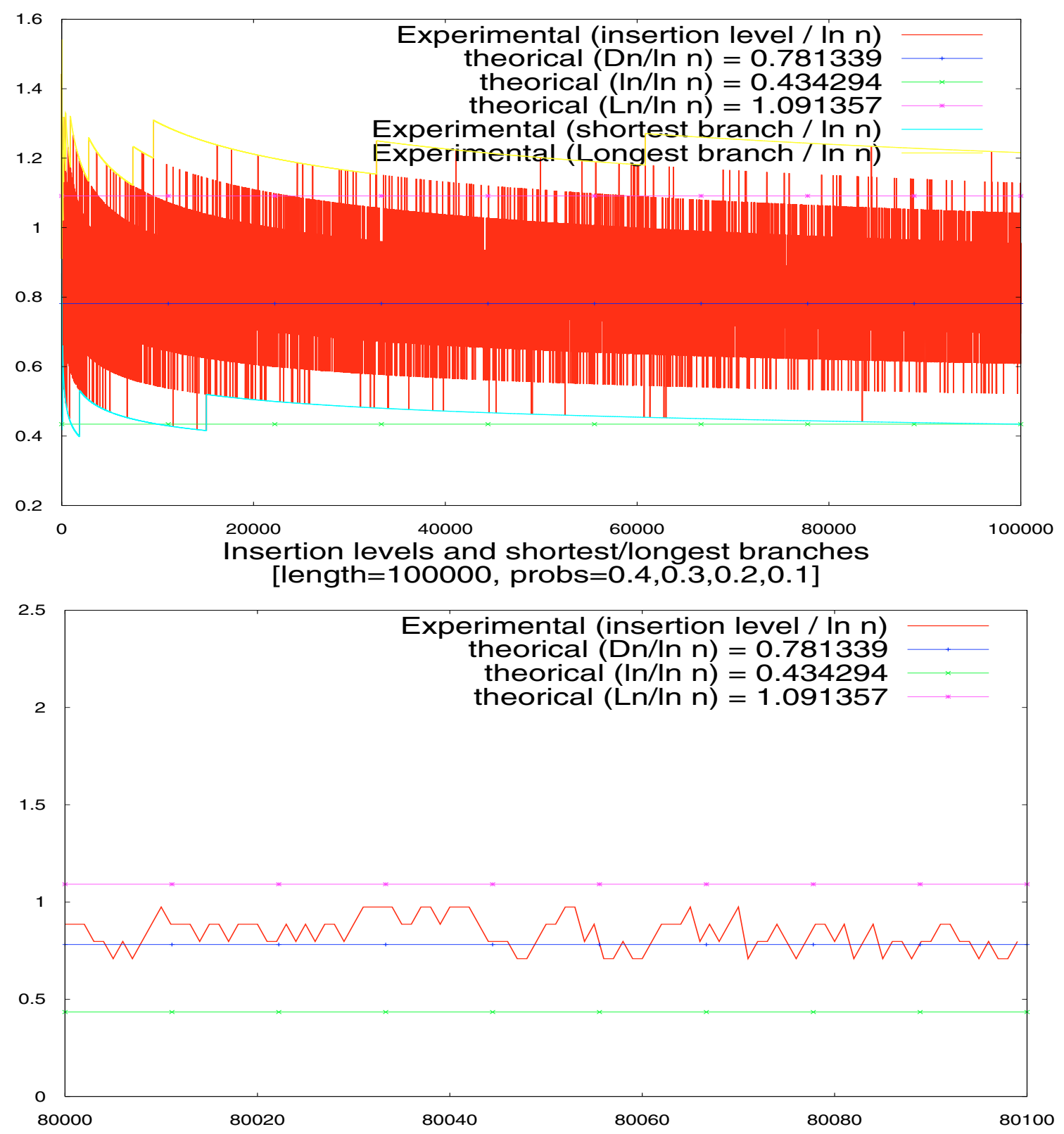

Figure 5. Simulations of one random sequence. Letters of the sequence are i.i.d. with respective probabilities $\left(p_{A}, p_{C}, p_{G}, p_{T}\right)=(0.4,0.3,0.2,0.1)$. On the $x$-axis, number $n$ of inserted letters; on the $y$-axis, normalized insertion depth $D_{n} / \ln n$ (oscillating curve), lengths of the shortest and of the longest branch (regular "under" and "upper envelops"). The horizontal lines correspond to the constant limits of these three random variables. 
Insertion levels and shortest/longest branches [length $=100000$, probs $=0.25,0.25,0.25,0.25]$
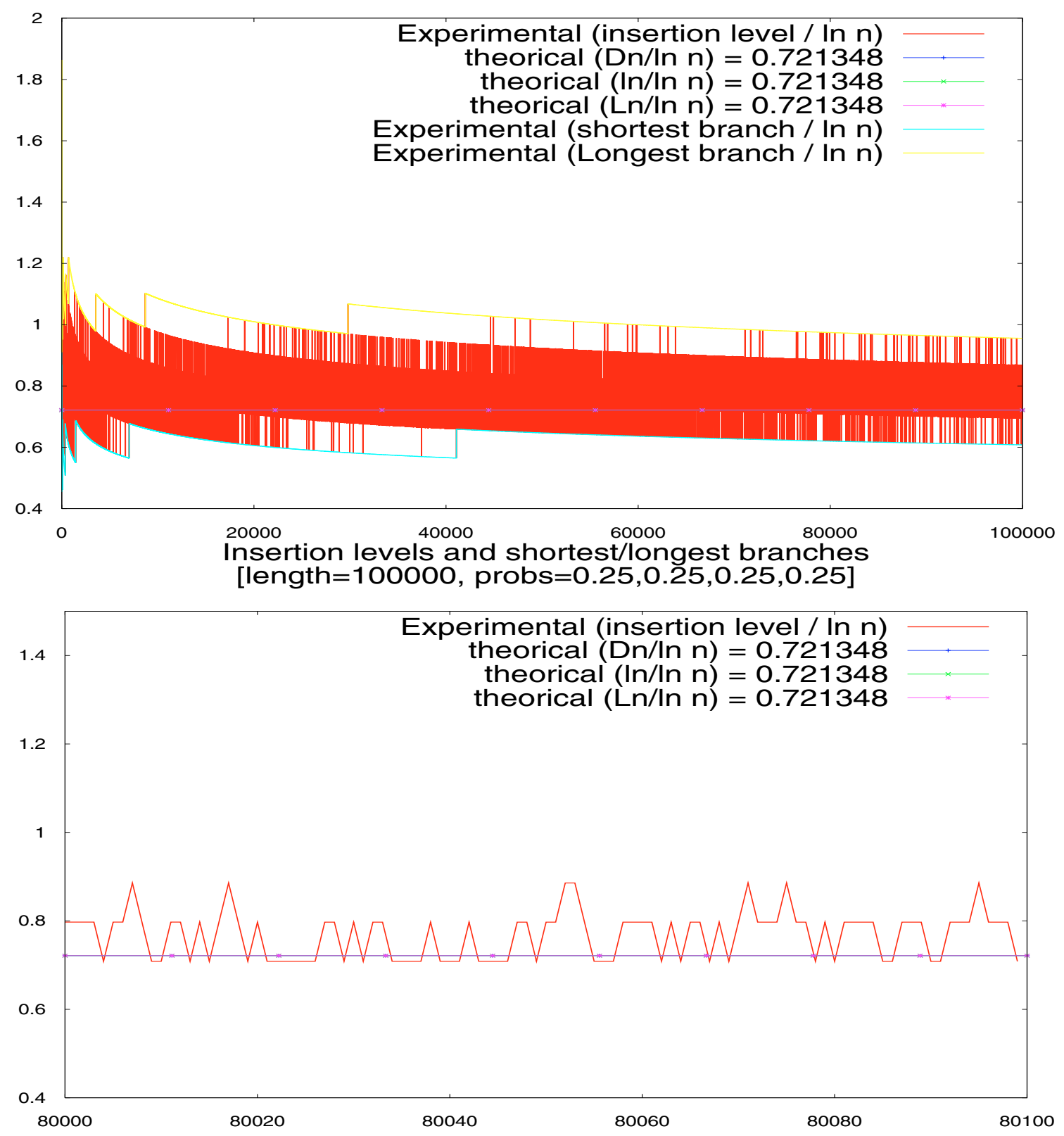

Figure 6. Simulations of one random sequence. Letters of the sequence are i.i.d. and equally likely distributed. On the $x$-axis, number $n$ of inserted letters; on the $y$-axis, normalized insertion depth $D_{n} / \ln n$ (oscillating curve), lengths of the shortest and of the longest branch (regular "under" and "upper envelops"). The horizontal line corresponds to the constant common limit of these three random variables. 


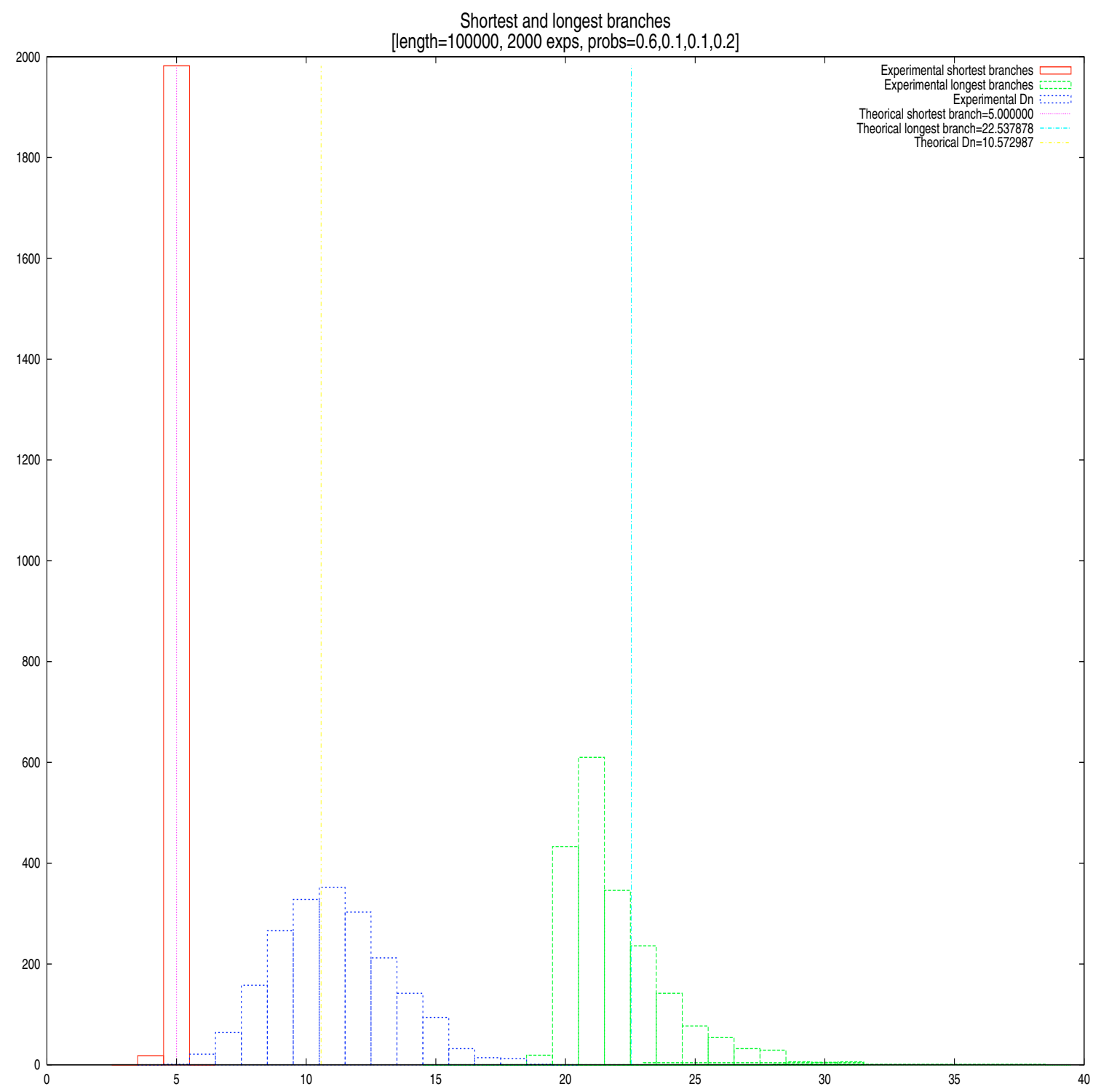

Figure 7. Simulations of 2000 sequences of 100,000 i.i.d. letters. On the left, histogram of shortest branches; in the middle, histogram of insertion depth of the last inserted word; on the right, histogram of longest branches. Vertical lines are their expected values, namely $\ln \left(10^{5}\right) \times \ell$ where $\ell$ respectively equals the limit of $\ell_{n} / \ln n, D_{n} / \ln n$ and $\mathcal{L}_{n} / \ln n$.

insertion depth of the last inserted word, lengths of the longest branches. On the $y$-axis, number of occurences (histograms).

Proof of Lemma 3.2. Since $X_{n}(s)=k$ for $n=T_{k}(s)$ (see Eq. (4)), by monotonicity arguments, it is sufficient to prove that

$$
\frac{\ln T_{k}(s)}{k} \underset{\mathrm{k} \rightarrow \infty}{\stackrel{\text { a.s. }}{\longrightarrow}} h(s) \text {. }
$$


Let $\varepsilon_{r}(s) \stackrel{\text { def }}{=} Z_{r}(s)-\mathbb{E}\left[Z_{r}(s)\right]$, so that $T_{k}(s)$ admits the decomposition

$$
T_{k}(s)=\mathbb{E}\left[T_{k}(s)\right]+\sum_{r=1}^{k} \varepsilon_{r}(s)
$$

If $\left(M_{k}(s)\right)_{k}$ is the martingale defined by

$$
M_{k}(s) \stackrel{\text { def }}{=} \sum_{r=1}^{k} \varepsilon_{r}(s),
$$

taking the logarithm in the preceding equation leads to

$$
\ln T_{k}(s)=\ln \mathbb{E}\left[T_{k}(s)\right]+\ln \left(1+\frac{M_{k}(s)}{\mathbb{E}\left[T_{k}(s)\right]}\right)
$$

- It is shown in Robin and Daudin [27] that $\mathbb{E}\left[Z_{n}(s)\right]=1 / p\left(s^{(n)}\right)$ so that the sequence $\frac{1}{n} \ln \mathbb{E}\left[Z_{n}(s)\right]$ converges to $h(s)$ as $n$ tends to infinity $\left(h(s)\right.$ is defined by (10)). Since $\mathbb{E}\left[T_{k}(s)\right]=\sum_{r=1}^{k} \mathbb{E}\left[Z_{r}(s)\right]$ (see (5)), the equality

$$
\lim _{k \rightarrow \infty} \frac{1}{k} \ln \mathbb{E}\left[T_{k}(s)\right]=h(s)
$$

is a straightforward consequence of the following elementary result: if $\left(x_{k}\right)_{k}$ is a sequence of positive numbers such that $\lim _{k \rightarrow \infty} \frac{1}{k} \ln \left(x_{k}\right)=h>0$, then $\lim _{k \rightarrow \infty} \frac{1}{k} \ln \left(\sum_{r=1}^{k} x_{r}\right)=h$.

- The martingale $\left(M_{k}(s)\right)_{k}$ is square integrable; let $\left(\langle M(s)\rangle_{k}\right)_{k}$ be its increasing process, defined by

$$
\langle M(s)\rangle_{k} \stackrel{\text { def }}{=} \sum_{j=2}^{k} \mathbb{E}\left[\left(M_{j}-M_{j-1}\right)^{2} \mid \mathcal{F}_{j-1}\right]=\sum_{j=2}^{k} \mathbb{E}\left[\varepsilon_{j}(s)^{2} \mid \mathcal{F}_{j-1}\right] .
$$

Robin and Daudin [27] have shown that the variance of $Z_{r}(s)$ satisfies $\mathbb{V}\left[Z_{r}(s)\right] \leq 4 r / p\left(s^{(r)}\right)^{2}$, so that

$$
\langle M(s)\rangle_{k}=O\left(k e^{2 k h(s)}\right)
$$

One can thus apply the Law of Large Numbers for martingales (see Duflo [8] for a reference on the subject): for any $\alpha>0$,

$$
M_{k}(s)=O\left(\langle M(s)\rangle_{k}^{1 / 2}\left(\ln \langle M(s)\rangle_{k}\right)^{\frac{1+\alpha}{2}}\right) \quad \text { a.s. }
$$

Consequently,

$$
\frac{M_{k}(s)}{\mathbb{E}\left[T_{k}(s)\right]}=O\left(k^{1+\alpha / 2}\right) \quad \text { a.s. }
$$

which completes the proof of Lemma 3.2.

Proof of Theorem 3.1. It is inspired from Pittel [23]. Clearly the definition given in Equation (9) yields

$$
\ell_{n} \leq X_{n}\left(s_{+}\right) \quad \text { and } \quad \mathcal{L}_{n} \geq X_{n}\left(s_{-}\right)
$$

(definitions of $s_{+}$and $s_{-}$were given in (2)). Hence, by Lemma 3.2

$$
\limsup _{n \rightarrow \infty} \frac{\ell_{n}}{\ln n} \leq \frac{1}{h_{+}}, \quad \liminf _{n \rightarrow \infty} \frac{\mathcal{L}_{n}}{\ln n} \geq \frac{1}{h_{-}} \quad \text { a.s. }
$$


- Proof for $\ell_{n}$

For any integer $r$,

$$
\mathbb{P}\left(\ell_{n} \leq r-1\right) \leq \sum_{s^{(r)} \in \mathcal{A}^{r}} \mathbb{P}\left(X_{n}(s) \leq r-1\right) \leq \sum_{s^{(r)} \in \mathcal{A}^{r}} \mathbb{P}\left(T_{r}(s) \geq n\right),
$$

where the above sums are taken over the set $\mathcal{A}^{r}$ of words with length $r$ (for a proper meaning of this formula, one should replace $s$ by any infinite word having $s^{(r)}$ as prefix, in both occurences). We abuse of this notation from now on. Since the generating functions $\Phi\left(s^{j}, t\right)$ are defined for any $1 \leq t<\min \left\{\gamma^{-1}, 1+\kappa p\left(s^{(r)}\right)\right\}$ and $j \leq r$ (see Assertion i) in Prop. 2.2), each term of the sum (12) can be controlled by

$$
\mathbb{P}\left(T_{r}(s) \geq n\right) \leq t^{-n} \mathbb{E}\left[t^{T_{r}(s)}\right] \leq t^{-n} \prod_{j=1}^{r} \Phi\left(s^{(j)}, t\right) .
$$

In particular, bounding above all the overlapping functions $\mathbb{1}_{\left\{s_{j} \ldots s_{1}=s_{r} \ldots s_{r-j+1}\right\}}$ by 1 in (7), we deduce from (6) and from Assertion ii) of Proposition 2.2 that

$$
\mathbb{P}\left(T_{r}(s) \geq n\right) \leq t^{-n} \prod_{j=1}^{r}\left(1+(1-t)\left(\sum_{\nu=1}^{j} \frac{1}{t^{\nu} p\left(s^{(\nu)}\right)}+\frac{\kappa^{\prime}}{1-\gamma t}\right)\right)^{-1} .
$$

Let $0<\varepsilon<1$. There exists a constant $\left.c_{2} \in\right] 0,1[$ depending only on $\varepsilon$ such that

$$
p\left(s^{(j)}\right)>c_{2} \alpha^{j}, \quad \text { with } \quad \alpha \stackrel{\text { def }}{=} \exp \left(-\left(1+\varepsilon^{2}\right) h_{+}\right)
$$

(for the sake of brevity $c, c_{1}$ and $c_{2}$ denote different constants all along the text). We then have

$$
\mathbb{P}\left(T_{r}(s) \geq n\right) \leq t^{-n} \prod_{j=1}^{r}\left(1+(1-t)\left(\frac{1-(\alpha t)^{-j}}{c_{2}(\alpha t-1)}+\frac{\kappa^{\prime}}{1-\gamma t}\right)\right)^{-1} .
$$

Choosing $t=1+c_{2} \kappa \alpha^{r}$, Inequality (8) is valid if $r$ is large enough, so that

$$
\mathbb{P}\left(T_{r}(s) \geq n\right) \leq c t^{-n} \prod_{j=1}^{r}\left(1-\kappa \alpha^{r-j} \frac{\alpha^{j}-\left(1+c_{2} \kappa \alpha^{r}\right)^{-j}}{\alpha\left(1+c_{2} \kappa \alpha^{r}\right)-1}-\frac{\alpha^{r} c_{2} \kappa \kappa^{\prime}}{1-\gamma\left(1+c_{2} \kappa \alpha^{r}\right)}\right)^{-1} .
$$

Moreover since obviously

$$
\lim _{\substack{j, r \rightarrow \infty \\ j \leq r}} \frac{\alpha^{j}-\left(1+c_{2} \kappa \alpha^{r}\right)^{-j}}{\alpha\left(1+c_{2} \kappa \alpha^{r}\right)-1}=\frac{1}{1-\alpha}
$$

and $c_{2} \kappa \kappa^{\prime} /\left(1-\gamma\left(1+c_{2} \kappa \alpha^{r}\right)\right)$ is uniformly bounded in $r$, there exist two positive constants $\lambda$ and $L$ independent of $j$ and $r$ such that

$$
\mathbb{P}\left(T_{r}(s) \geq n\right) \leq\left(1+c_{2} \kappa \alpha^{r}\right)^{-n} L \prod_{j=1}^{r}\left(1-\lambda \alpha^{r-j}\right)^{-1} .
$$

In addition, the product can be bounded above by

$$
\prod_{j=1}^{r}\left(1-\lambda \alpha^{r-j}\right)^{-1} \leq \prod_{j=0}^{\infty}\left(1-\lambda \alpha^{j}\right)^{-1}=R<\infty .
$$


Consequently,

$$
\mathbb{P}\left(T_{r}(s) \geq n\right) \leq L R\left(1+c_{2} \kappa \alpha^{r}\right)^{-n} .
$$

For $r=\left\lfloor(1-\varepsilon) \frac{\ln n}{h_{+}}\right\rfloor$and $\varepsilon$ small enough, there exists a constant $R^{\prime}$ such that

$$
\mathbb{P}\left(T_{r}(s) \geq n\right) \leq R^{\prime} \exp \left(-c_{2} \kappa n^{\theta}\right),
$$

where $\theta=\varepsilon-\varepsilon^{2}+\varepsilon^{3}>0$. We then deduce from (12) that

$$
\mathbb{P}\left(\ell_{n} \leq r-1\right) \leq 4^{r} R^{\prime} \exp \left(-c_{2} \kappa n^{\theta}\right)
$$

which is the general term of a convergent series. Borel-Cantelli lemma applies so that

$$
\liminf _{n \rightarrow \infty} \frac{\ell_{n}}{\ln n} \geq \frac{1}{h_{+}} \quad \text { a.s. }
$$

- Proof for $\mathcal{L}_{n}$

To complete the proof, one needs to show that

$$
\limsup _{n \rightarrow \infty} \frac{\mathcal{L}_{n}}{\ln n} \leq \frac{1}{h_{-}} \quad \text { a.s. }
$$

Again, since $X_{n}(s)=k$ for $n=T_{k}(s)$, by monotonicity arguments it suffices to show that

$$
\liminf _{k \rightarrow \infty} \min _{s^{(k)} \in \mathcal{A}^{k}} \frac{\ln T_{k}(s)}{k} \geq h_{-} \quad \text { a.s. }
$$

(notations of (12)).

Let $0<\varepsilon<1$. As in the previous proof for the shortest branches, it suffices to bound above

$$
\mathbb{P}\left(\min _{s^{(k)} \in \mathcal{A}^{k}} T_{k}(s)<e^{k h_{-}(1-\varepsilon)}\right)
$$

by the general term of a convergent series to apply Borel-Cantelli lemma. Obviously,

$$
\mathbb{P}\left(\min _{s^{(k)} \in \mathcal{A}^{k}} T_{k}(s)<e^{k h_{-}(1-\varepsilon)}\right) \leq \sum_{s^{(k)} \in \mathcal{A}^{k}} \mathbb{P}\left(T_{k}(s)<e^{k h_{-}(1-\varepsilon)}\right) .
$$

If $t$ is any real number in $] 0,1\left[\right.$ and if $n \stackrel{\text { def }}{=} \exp \left(k h_{-}(1-\varepsilon)\right)$,

$$
\mathbb{P}\left(T_{k}(s)<e^{k h_{-}(1-\varepsilon)}\right)=\mathbb{P}\left(t^{T_{k}(s)}>t^{n}\right)
$$

and the decomposition (5), together with the independence of the $Z_{r}(s)$ for $1 \leq r \leq k$, yield

$$
\mathbb{P}\left(t^{T_{k}(s)}>t^{n}\right) \leq t^{-n} \prod_{r=1}^{k} \mathbb{E}\left[t^{Z_{r}(s)}\right] .
$$

The proof consists in bounding above

$$
\sum_{s^{(k)} \in \mathcal{A}^{k}} t^{-n} \prod_{r=1}^{k} \mathbb{E}\left[t^{Z_{r}(s)}\right]
$$


by the general term of a convergent series, taking $t$ of the form

$$
t \stackrel{\text { def }}{=}(1+c / n)^{-1}
$$

so that the sequence $\left(t^{n}\right)_{n}$ is bounded.

The generating function of $Z_{r}(s)$ is given by Robin and Daudin [27] and strongly depends on the overlapping structure of the word $s^{(r)}$. As $0<t<1$, this function is well defined at $t$ and is given by (see Assertion i) of Prop. 2.2)

$$
\mathbb{E}\left[t^{Z_{r}(s)}\right]=1-\frac{(1-t)}{t^{r} p\left(s^{(r)}\right)\left(\gamma_{r}(t)+(1-t) \delta_{r}\left(t^{-1}\right)\right)},
$$

where $\gamma_{r}(t)$ and $\delta_{r}(t)$ are defined in (7). Moreover, from Assertion ii) of Proposition 2.2, it is obvious that there exists a constant $\theta$ independent of $r$ and $s$ such that,

$$
\gamma_{r}(t) \leq 1+\theta(1-t)
$$

Besides, by elementary change of variable, one has successively

$$
\begin{aligned}
t^{r} p\left(s^{(r)}\right) \delta_{r}\left(t^{-1}\right) & =\sum_{m=1}^{r} \mathbb{1}_{\left\{s_{r} \ldots s_{r-m+1}=s_{m} \ldots s_{1}\right\}} \frac{t^{r} p\left(s^{(r)}\right)}{t^{m} p\left(s^{(m)}\right)} \\
& =\sum_{m=1}^{r} \mathbb{1}_{\left\{s_{r} \ldots s_{m}=s_{r-m+1} \ldots s_{1}\right\}} t^{m-1} \frac{p\left(s^{(r)}\right)}{p\left(s^{(r-m+1)}\right)} \\
& =\sum_{m=1}^{r} \mathbb{1}_{\left\{s_{r} \ldots s_{m}=s_{r-m+1} \ldots s_{1}\right\}} t^{m-1} \frac{p\left(s^{(m)}\right)}{p\left(s_{m}\right)}
\end{aligned}
$$

When $m$ is large enough, $h_{-}$'s definition implies that

$$
p\left(s^{(m)}\right) \leq \beta^{m}, \quad \text { where } \quad \beta \stackrel{\text { def }}{=} \exp \left(-\left(1-\varepsilon^{2}\right) h_{-}\right),
$$

so that there exists positive constants $\rho$ and $c$ such that, for any $r$,

$$
p\left(s^{(r)}\right) \leq c \beta^{r} \quad \text { and } \quad t^{r} p\left(s^{(r)}\right) \delta_{r}\left(t^{-1}\right) \leq 1+\rho \sum_{m=2}^{r} \mathbb{1}_{\left\{s_{r} \ldots s_{m}=s_{r-m+1} \ldots s_{1}\right\}} \beta^{m} .
$$

Thus Formula (14) with inequalities (15) and (16) yield, for any $r \leq k$,

$$
\mathbb{E}\left[t^{Z_{r}(s)}\right] \leq 1-\frac{1}{c \beta^{r}\left(\frac{1}{1-t}+\theta\right)+1+q_{k}(s)}
$$

where $q_{k}(s)$, that depends on the overlapping structure of $s^{(k)}$, is defined by

$$
q_{k}(s) \stackrel{\text { def }}{=} \rho \max _{2 \leq r \leq k} \sum_{m=2}^{r} \mathbb{1}_{\left\{s_{r} \ldots s_{m}=s_{r-m+1} \ldots s_{1}\right\}} \beta^{m} .
$$

Note that whatever the overlapping structure is, $q_{k}(s)$ is controlled by

$$
0 \leq q_{k}(s) \leq \frac{\rho}{1-\beta} .
$$


Thus,

$$
\prod_{r=1}^{k} \mathbb{E}\left[t^{Z_{r}(s)}\right] \leq \exp \left[-\sum_{r=1}^{k} \ln \left(1-\frac{1}{c \beta^{r}\left((1-t)^{-1}+\theta\right)+1+q_{k}(s)}\right)^{-1}\right] .
$$

Since the function $x \mapsto \ln 1 /(1-x)$ is increasing, comparing this sum with an integral and after the change of variable $y=c \beta^{x}\left((1-t)^{-1}+\theta\right)$, one obtains

$$
\prod_{r=1}^{k} \mathbb{E}\left[t^{Z_{r}(s)}\right] \leq \exp \left[-\frac{1}{\ln \beta^{-1}} \int_{c \beta^{k}\left((1-t)^{-1}+\theta\right)}^{c\left((1-t)^{-1}+\theta\right)} \ln \left(1-\frac{1}{y+1+q_{k}(s)}\right)^{-1} \frac{\mathrm{d} y}{y}\right] .
$$

This integral is convergent in a neighbourhood of $+\infty$, hence there exists a constant $C$, independent of $k$ and $s$ such that

$$
\prod_{r=1}^{k} \mathbb{E}\left[t^{Z_{r}(s)}\right] \leq C \exp \left[-\frac{1}{\ln \beta^{-1}} \int_{c \beta^{k}\left((1-t)^{-1}+\theta\right)}^{+\infty} \ln \left(1-\frac{1}{y+1+q_{k}(s)}\right)^{-1} \frac{\mathrm{d} y}{y}\right] .
$$

The classical dilogarithm $\operatorname{Li}_{2}(z)=\sum_{k \geq 1} z^{k} / k^{2}$, analytically continued to the complex plane slit along the ray $\left[1,+\infty\left[\right.\right.$, satisfies $\frac{\mathrm{d}}{\mathrm{d} y} \operatorname{Li}_{2}\left(-\frac{v}{y}\right)=\frac{1}{y} \ln (1+v / y)$. This leads to the formula

$$
\int_{a_{k}}^{+\infty} \ln \left(1-\frac{1}{y+1+q_{k}(s)}\right)^{-1} \frac{\mathrm{d} y}{y}=\operatorname{Li}_{2}\left(-\frac{q_{k}(s)}{a_{k}}\right)-\operatorname{Li}_{2}\left(-\frac{1+q_{k}(s)}{a_{k}}\right)
$$

with the notation $a_{k}=c \beta^{k}\left((1-t)^{-1}+\theta\right)$. Choosing $t=(1+c / n)^{-1}$ yields readily

$$
a_{k} \underset{k \rightarrow+\infty}{\sim} \exp \left(-k h_{-}\left(\varepsilon-\varepsilon^{2}\right)\right)
$$

Moreover, in a neighbourhood of $-\infty$,

$$
\operatorname{Li}_{2}(x)=-\frac{1}{2} \ln ^{2}(-x)-\zeta(2)+O\left(\frac{1}{x}\right),
$$

( $\zeta$ is Riemann's function, see for instance Abramowitz and Stegun [1]) and the function $\operatorname{Li}_{2}(x)+\frac{1}{2} \ln ^{2}(-x)$ is non-decreasing on $]-\infty, 0[$, so that

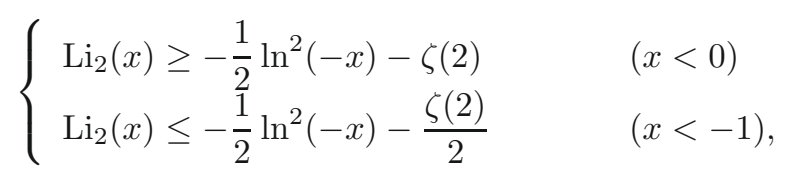

noting that $\operatorname{Li}_{2}(-1)=-\frac{\zeta(2)}{2}$. Hence, if $k$ is such that $a_{k}<1$,

$$
\int_{a_{k}}^{+\infty} \ln \left(1-\frac{1}{y+1+q_{k}(s)}\right)^{-1} \frac{\mathrm{d} y}{y} \geq \operatorname{Li}_{2}\left(-\frac{q_{k}(s)}{a_{k}}\right)+\frac{1}{2} \ln ^{2}\left(a_{k}\right)+\frac{\zeta(2)}{2}
$$

with $\ln a_{k}$ being asymptotically proportional to $k$ because of (20). Thus, the behaviour of the integral in (19) as $k$ tends to $+\infty$ depends on the asymptotics of $q_{k}(s)$.

Let $z_{k} \stackrel{\text { def }}{=} \exp (-\sqrt{k})$. The end of the proof consists, for a given $k$, in splitting the sum (13) into prefixes $s^{(k)}$ that respectively satisfy $q_{k}(s)<\exp (-\sqrt{k})$ or $q_{k}(s) \geq \exp (-\sqrt{k})$. These two cases correspond to words that respectly have few or many overlapping patterns. The choice $z_{k}=\exp (-\sqrt{k})$ is arbitrary and many other sequences could have been taken provided that they converge to zero with a speed of the form $\exp [-o(k)]$. 
First let us consider the case of prefixes $s^{(k)}$ such that $q_{k}(s)<\exp (-\sqrt{k})$. For such words, (22) and (23) imply that

$$
\int_{a_{k}}^{+\infty} \ln \left(1-\frac{1}{y+1+q_{k}(s)}\right)^{-1} \frac{\mathrm{d} y}{y} \geq-\frac{1}{2} \ln ^{2}\left(\frac{z_{k}}{a_{k}}\right)+\frac{1}{2} \ln ^{2}\left(a_{k}\right)-\frac{\zeta(2)}{2},
$$

the second member of this inequality being, as $k$ tends to infinity, of the form

$$
k \sqrt{k} h_{-}\left(\varepsilon-\varepsilon^{2}\right)+O(k) .
$$

Consequently,

$$
\prod_{r=1}^{k} \mathbb{E}\left[t^{Z_{r}(s)}\right] \leq \exp \left[-\frac{\varepsilon}{1+\varepsilon} k^{3 / 2}+O(k)\right] .
$$

There are $4^{k}$ words of length $k$, hence very roughly, by taking the sum over the prefixes $s^{(k)}$ such that $q_{k}(s)<z_{k}$, and since $t^{-n}$ is bounded, the contribution of these prefixes to the sum (13) satisfies

$$
\sum_{s^{(k)} \in \mathcal{A}^{k}, q_{k}(s)<z_{k}} t^{-n} \prod_{r=1}^{k} \mathbb{E}\left[t^{Z_{r}(s)}\right] \leq 4^{k} \exp \left[-\frac{\varepsilon}{1+\varepsilon} k^{3 / 2}+O(k)\right],
$$

which is the general term of a convergent series.

It remains to study the case $q_{k}(s) \geq z_{k}$. For such words, let us only consider the inequalities (18) and (19) that lead to

$$
\prod_{r=1}^{k} \mathbb{E}\left[t^{Z_{r}(s)}\right] \leq C \exp \left[-\frac{1}{\ln \beta^{-1}} \int_{a_{k}}^{+\infty} \ln \left(1-\frac{1}{y+1+\rho(1-\beta)^{-1}}\right)^{-1} \frac{\mathrm{d} y}{y}\right] .
$$

Since $x \leq \log (1-x)^{-1}$, after some work of integration,

$$
\prod_{r=1}^{k} \mathbb{E}\left[t^{Z_{r}(s)}\right] \leq \exp \left(-\frac{\varepsilon}{1+\varepsilon} k+o(k)\right)
$$

The natural question arising now is: how many words $s^{(k)}$ are there, such that $q_{k}(s) \geq z_{k}$ ? Let us define

$$
E_{k} \stackrel{\text { def }}{=}\left\{s^{(k)}, q_{k}(s) \geq e^{-\sqrt{k}}\right\}
$$

The definition of $q_{k}(s)$ implies clearly that

$$
E_{k} \subseteq\left\{s^{(k)}, \exists r \leq k, \rho \sum_{m=2}^{r} \mathbb{1}_{\left\{s_{r} \ldots s_{m}=s_{r-m+1} \ldots s_{1}\right\}} \beta^{m} \geq e^{-\sqrt{k}}\right\} .
$$

For any $r \leq k$ and $x>0$, let us define the set

$$
S_{r}(x) \stackrel{\text { def }}{=}\left\{s^{(k)}, \sum_{m=2}^{r} \mathbb{1}_{\left\{s_{r} \ldots s_{m}=s_{r-m+1} \ldots s_{1}\right\}} \beta^{m}<x\right\} .
$$

For any $\ell \in\{2, \ldots, r\}$, one has the following inclusion

$$
\bigcap_{m=2}^{\ell}\left\{s^{(k)}, \mathbb{1}_{\left\{s_{r} \ldots s_{m}=s_{r-m+1} \ldots s_{1}\right\}}=0\right\} \subset S_{r}\left(\frac{\beta^{\ell+1}}{1-\beta}\right) .
$$


If the notation $B^{c}$ denotes the complementary set of $B$ in $\mathcal{A}^{k}$,

$$
S_{r}^{c}\left(\frac{\beta^{\ell+1}}{1-\beta}\right) \subset \bigcup_{m=2}^{\ell}\left\{s^{(k)}, \mathbb{1}_{\left\{s_{r} \ldots s_{m}=s_{r-m+1} \ldots s_{1}\right\}}=1\right\} .
$$

Since $e^{-\sqrt{k}}=\rho \beta^{\ell+1}(1-\beta)^{-1}$ for $\ell \stackrel{\text { def }}{=} \sqrt{k} / \ln \left(\beta^{-1}\right)+\ln \left(\rho^{-1}(1-\beta)\right) / \ln \beta$,

$$
E_{k} \subset \bigcup_{r=1}^{k} \bigcup_{m=2}^{\lfloor\ell\rfloor+1}\left\{s^{(k)}, \mathbb{1}_{\left\{s_{r} \ldots s_{m}=s_{r-m+1} \ldots s_{1}\right\}}=1\right\},
$$

so that the number of words $s^{(k)}$ such that $q_{k}(s) \geq z_{k}$ is bounded above by

$$
\# E_{k} \leq \sum_{r=1}^{k} \sum_{m=2}^{\lfloor\ell\rfloor+1} 4^{m-1} \in O\left(k 4^{\sqrt{k} / \ln \left(\beta^{-1}\right)}\right)
$$

Putting (24) and (25) together is sufficient to show that the contribution of prefixes $s^{(k)}$ such that $q_{k}(s) \geq z_{k}$ to the sum (13), namely

$$
\sum_{s^{(k)} \in \mathcal{A}^{k}, q_{k}(s) \geq z_{k}} t^{-n} \prod_{r=1}^{k} \mathbb{E}\left[t^{Z_{r}(s)}\right],
$$

is the general term of a convergent series too.

Finally, the whole sum (13) is the general term of a convergent series, which completes the proof of the inequality

$$
\limsup _{n \rightarrow \infty} \frac{\mathcal{L}_{n}}{\ln n} \leq \frac{1}{h_{-}} \quad \text { a.s. }
$$

\section{INSERTION DEPTH}

This section is devoted to the asymptotic behaviour of the insertion depth denoted by $D_{n}$ and to the length of a path randomly and uniformly chosen denoted by $M_{n}$ (see Sect. 2). $D_{n}$ is defined as the length of the path leading to the node where $W(n)$ is inserted. In other words, $D_{n}$ is the amount of digits to be checked before the position of $W(n)$ is found. Theorem 3.1 immediately implies a first asymptotic result on $D_{n}$. Indeed, $D_{n}=\ell_{n}$ whenever $\ell_{n+1}>\ell_{n}$, which happens infinitely often a.s., since $\lim _{n \rightarrow \infty} \ell_{n}=\infty$ a.s. Hence,

$$
\liminf _{n \rightarrow \infty} \frac{D_{n}}{\ln n}=\liminf _{n \rightarrow \infty} \frac{\ell_{n}}{\ln n}=\frac{1}{h_{+}} \quad \text { a.s. }
$$

Similarly, $D_{n}=\mathcal{L}_{n}$ whenever $\mathcal{L}_{n+1}>\mathcal{L}_{n}$, and hence

$$
\limsup _{n \rightarrow \infty} \frac{D_{n}}{\ln n}=\limsup _{n \rightarrow \infty} \frac{\mathcal{L}_{n}}{\ln n}=\frac{1}{h_{-}} \quad \text { a.s. }
$$

Theorem 4.1 states full convergence in probability of these random variables to the constant $1 / h$.

Theorem 4.1.

$$
\frac{D_{n}}{\ln n} \underset{\mathrm{n} \rightarrow \infty}{\stackrel{\mathrm{P}}{\longrightarrow}} \frac{1}{h} \quad \text { and } \quad \lim _{n \rightarrow \infty} \frac{M_{n}}{\ln n} \underset{\mathrm{n} \rightarrow \infty}{\stackrel{\mathrm{P}}{\longrightarrow}} \frac{1}{h} .
$$


Remark 4.2. For an i.i.d. sequence $U=U_{1} U_{2} \ldots$, in the case when the random variables $U_{i}$ are not uniformly distributed in $\{A, C, G, T\}$, both relations stated just before Theorem 4.1 imply that $\frac{D_{n}}{\ln n}$ does not converge a.s. because

$$
\limsup _{n \rightarrow \infty} \frac{D_{n}}{\ln n}=\frac{1}{h_{-}}>\frac{1}{h_{+}}=\liminf _{n \rightarrow \infty} \frac{D_{n}}{\ln n} .
$$

Proof of Theorem 4.1. It suffices to consider $D_{n}$ since, by definition of $M_{n}$,

$$
\mathbb{P}\left(M_{n}=r\right)=\frac{1}{n} \sum_{\nu=1}^{n} \mathbb{P}\left(D_{\nu}=r\right)
$$

Let $\varepsilon>0$. To prove Theorem 4.1 , we get the convergence $\lim _{n \rightarrow \infty} \mathbb{P}\left(A_{n}\right)=0$, where

$$
A_{n} \stackrel{\text { def }}{=}\left\{U \in \mathcal{A}^{\mathbb{N}},\left|\frac{D_{n}}{\ln n}-\frac{1}{h}\right| \geq \frac{\varepsilon}{h}\right\}
$$

by using the obvious decomposition

$$
\mathbb{P}\left(A_{n}\right)=\mathbb{P}\left(\frac{D_{n}}{\ln n} \geq \frac{1+\varepsilon}{h}\right)+\mathbb{P}\left(\frac{D_{n}}{\ln n} \leq \frac{1-\varepsilon}{h}\right) .
$$

- Because of $X_{n}$ 's definition (3),

$$
D_{n}=X_{n-1}(W(n))+1
$$

so that the duality (4) between $X_{n}(s)$ and $T_{k}(s)$ implies that

$$
\mathbb{P}\left(\frac{D_{n}}{\ln n} \geq \frac{1+\varepsilon}{h}\right) \leq \mathbb{P}\left(X_{n-1}(W(n)) \geq k-1\right) \leq \mathbb{P}\left(T_{k-1}(W(n)) \leq n-1\right)
$$

with $k \stackrel{\text { def }}{=}\left\lfloor\frac{1+\varepsilon}{h} \ln n\right\rfloor$. Furthermore,

$$
\mathbb{P}\left(T_{k-1}(W(n)) \leq n-1\right) \leq \mathbb{P}\left(\left\{T_{k-1}(W(n)) \leq n-1\right\} \cap B_{n, k_{0}}\right)+\mathbb{P}\left(B_{n, k_{0}}^{c}\right)
$$

where $B_{n, k_{0}}$ is defined, for any $k_{0} \leq n$, by

$$
B_{n, k_{0}} \stackrel{\text { def }}{=} \bigcap_{k_{0} \leq j \leq n}\left\{U \in \mathcal{A}^{\mathbb{N}},\left|\frac{1}{j} \ln \left(\frac{1}{p\left(W(n)^{(j)}\right)}\right)-h\right| \leq \varepsilon^{2} h\right\} .
$$

Since the sequence $U$ is stationary, $\mathbb{P}\left(W(n)^{(j)}\right)=\mathbb{P}\left(U^{(j)}\right)$ so that the Ergodic theorem implies

$$
\lim _{j \rightarrow \infty} \frac{1}{j} \ln \left(\frac{1}{p\left(W(n)^{(j)}\right)}\right)=h \quad \text { a.s. }
$$

which leads to $\mathbb{P}\left(B_{n, k_{0}}\right) \geq 1-\eta$ for all $\eta>0$, when both $k_{0}$ and $n$ are large enough. If $\mathcal{S}_{n, k_{0}}$ denotes the set of words

$$
\mathcal{S}_{n, k_{0}} \stackrel{\text { def }}{=}\left\{s^{(n)} \in \mathcal{A}^{n}, \forall j \in\left\{k_{0}, \ldots, n\right\}\left|\frac{1}{j} \ln \left(\frac{1}{p\left(s^{(j)}\right)}\right)-h\right| \leq \varepsilon^{2} h\right\},
$$


when $k_{0}$ and $n$ are large enough,

$$
\begin{aligned}
\mathbb{P}\left(T_{k-1}(W(n)) \leq n-1\right) & \leq \sum_{s^{(n)} \in \mathcal{S}_{n, k_{0}}} \mathbb{P}\left(W(n)^{(n)}=s^{(n)}, T_{k-1}(s) \leq n-1\right)+\eta \\
& \leq \sum_{s^{(n)} \in \mathcal{S}_{n, k_{0}}} \mathbb{P}\left(T_{k-1}(s) \leq n-1\right)+\eta
\end{aligned}
$$

Such a probability has already been bounded above at the end of Theorem 3.1's proof; similarly,

$$
\sum_{s^{(n)} \in \mathcal{S}_{n, k_{0}}} \mathbb{P}\left(T_{k-1}(s) \leq n-1\right)=O\left(n \exp \left(-\frac{\varepsilon}{1+\varepsilon} n+\frac{\ln 4}{\left(1-\varepsilon^{2}\right) h} \sqrt{n}\right)\right)
$$

so that (26) and (27) show that $\mathbb{P}\left(\frac{D_{n}}{\ln n} \geq \frac{1+\varepsilon}{h}\right)$ tends to zero when $n$ goes off to infinity.

- Our argument showing that $\mathbb{P}\left(\frac{D_{n}}{\ln n} \leq \frac{1-\varepsilon}{h}\right)$ tends to zero when $n$ tends to infinity is similar. If now $k \stackrel{\text { def }}{=}\left\lfloor\frac{1-\varepsilon}{h} \ln n\right\rfloor$,

$$
\mathbb{P}\left(\frac{D_{n}}{\ln n} \leq \frac{1-\varepsilon}{h}\right) \leq \mathbb{P}\left(X_{n-1}(W(n)) \leq k-1\right)=\mathbb{P}\left(T_{k}(W(n)) \geq n\right),
$$

so that

$$
\mathbb{P}\left(\frac{D_{n}}{\ln n} \leq \frac{1-\varepsilon}{h}\right) \leq \mathbb{P}\left(\left\{T_{k}(W(n)) \geq n\right\} \cap B_{n, k_{0}}\right)+\mathbb{P}\left(B_{n, k_{0}}^{c}\right) .
$$

As before, $\mathbb{P}\left(B_{n, k_{0}}^{c}\right)=0$ when $k_{0}$ and $n$ are large enough and

$$
\begin{aligned}
\mathbb{P}\left(T_{k}(W(n)) \geq n\right) & \leq \sum_{s^{(n)} \in \mathcal{S}_{n, k_{0}}} \mathbb{P}\left(W(n)^{(n)}=s^{(n)}, T_{k}(s) \geq n\right) \\
& \leq \sum_{s^{(n)} \in \mathcal{S}_{n, k_{0}}} \mathbb{P}\left(T_{k}(s) \geq n\right) .
\end{aligned}
$$

Like in the proof of Theorem 3.1, one shows that

$$
\sum_{s^{(n)} \in \mathcal{S}_{n, k_{0}}} \mathbb{P}\left(T_{k}(s) \geq n\right)=O\left(4^{n} \exp \left(-\kappa n^{\theta} / 2\right)\right)
$$

which implies that $\mathbb{P}\left(\frac{D_{n}}{\ln n} \leq \frac{1-\varepsilon}{h}\right)$ tends to zero when $n$ tends to infinity. The proof of Theorem 4.1 is complete.

\section{A. Domain of Definition of the Generating function $\Phi\left(s^{(r)}, t\right)$}

\section{A.1. Proof of Assertion ii)}

There exists a function $K\left(s_{1}, s_{r}, m\right)$ uniformly bounded by the constant

$$
\left.K \stackrel{\text { def }}{=} \sup _{s_{1}, s_{r}, m} \mid K\left(s_{1}, s_{r}, m\right)\right) \mid
$$

such that

$$
Q^{m}\left(s_{1}, s_{r}\right)-p\left(s_{r}\right)=K\left(s_{1}, s_{r}, m\right) \gamma^{m}
$$


where $\gamma$ is the second eigenvalue of the transition matrix. Consequently,

$$
\begin{aligned}
\left|\gamma_{r}(t)-1\right| & =\left|\frac{1-t}{t p\left(s_{r}\right)} \sum_{m \geq 1} K\left(s_{1}, s_{r}, m\right)(\gamma t)^{m}\right| \\
& \leq \frac{\gamma K}{\min _{u} p(u)} \frac{|1-t|}{1-\gamma|t|} .
\end{aligned}
$$

Hence Assertion ii) holds with $\kappa^{\prime} \stackrel{\text { def }}{=} \gamma K / \min _{u} p(u)$.

\section{A.2. Proof of Assertion i)}

On the unit disc $|t|<1$, the series

$$
S(t) \stackrel{\text { def }}{=} \frac{1}{t} \sum_{m \geq 1} Q^{m}\left(s_{1}, s_{r}\right) t^{m}
$$

is convergent and one has the decomposition

$$
\frac{1-t}{p\left(s_{r}\right) t} \sum_{m \geq 1} Q^{m}\left(s_{1}, s_{r}\right) t^{m}=1+\frac{1-t}{p\left(s_{r}\right) t} \sum_{m \geq 1}\left[Q^{m}\left(s_{1}, s_{r}\right)-p\left(s_{r}\right)\right] t^{m} .
$$

The function

$$
\sum_{m \geq 1}\left[Q^{m}\left(s_{1}, s_{r}\right)-p\left(s_{r}\right)\right] t^{m}
$$

is analytically continuable to the domain $\gamma|t|<1$, and then the series

$$
\frac{1-t}{t p\left(s_{r}\right)} \sum_{m \geq 1} Q^{m}\left(s_{1}, s_{r}\right) t^{m}
$$

converges on the same domain. One has to determine the zeroes of

$$
\begin{aligned}
D(t) \stackrel{\text { def }}{=} & p\left(s^{(r)}\right) t^{r}+\frac{(1-t) p\left(s^{(r)}\right) t^{r}}{p\left(s_{r}\right) t} \sum_{z \geq 1} t^{z}\left[Q^{z}\left(s_{1}, s_{r}\right)-p\left(s_{r}\right)\right] \\
& +(1-t)\left[1+\sum_{j=2}^{r} t^{j-1} \frac{p\left(s^{(j)}\right)}{p\left(s_{j}\right)} \mathbb{1}_{\left\{s_{r} \ldots s_{j}=s_{r-j+1} \ldots s_{1}\right\}}\right] .
\end{aligned}
$$

Assuming that some $0<t<1$ were a real root of $D(t)$, then

$$
\begin{aligned}
0 & <\frac{(1-t) p\left(s^{(r)}\right) t^{r}}{p\left(s_{r}\right) t} \sum_{z \geq 1} t^{z} Q^{z}\left(s_{1}, s_{r}\right) \\
& =(t-1)\left[1+\sum_{j=2}^{r} t^{j-1} \frac{p\left(s^{(j)}\right)}{p\left(s_{j}\right)} \mathbb{1}_{\left\{s_{r} \ldots s_{j}=s_{r-j+1} \ldots s_{1}\right\}}\right]<0 .
\end{aligned}
$$


It is thus obvious that there are no real root of $D(t)$ in $] 0,1[$. Moreover, one can readily check that 0 and 1 are not zeroes of $D(t)$. We now look for a root of the form $t=1+\varepsilon$ with $\varepsilon>0$. Such an $\varepsilon$ satisfies

$$
\varepsilon=\frac{(1+\varepsilon)^{r} p\left(s^{(r)}\right)\left(1-\frac{\varepsilon}{p\left(s_{r}\right)(1+\varepsilon)} \sum_{z \geq 1} t^{z}\left[Q^{z}\left(s_{1}, s_{r}\right)-p\left(s_{r}\right)\right]\right)}{1+\sum_{j=2}^{r}(1+\varepsilon)^{j-1} \frac{p\left(s^{(j)}\right)}{p\left(s_{j}\right)} \mathbb{1}_{\left\{s_{r} \ldots s_{j}=s_{r-j+1} \ldots s_{1}\right\}}},
$$

so that

$$
\varepsilon \geq \kappa p\left(s^{(r)}\right)
$$

This implies that $\Phi\left(s^{(r)}, t\right)$ is at least defined on $\left[0,1+\kappa p\left(s^{(r)}\right)[\right.$. This implies the result.

\section{REFERENCES}

[1] M. Abramowitz and I.A. Stegun, Handbook of mathematical functions with formulas, graphs, and mathematical tables, National Bureau of Standards Applied Mathematics Series 55. For sale by the superintendent of Documents, U.S. Government Printing Office, Washington, D.C. (1964).

[2] D. Aldous and P. Shields, A diffusion limit for a class of randomly-growing binary search trees. Probab. Theory Related Fields 79 (1998) 509-542.

[3] J.S. Almeida, J.A. Carriço, A. Maretzek, P.A. Noble and M. Fletcher, Analysis of genomic sequences by Chaos Game Representation. Bioinformatics 17 (2001) 429-437.

[4] G. Blom and D. Thorburn, How many random digits are required until given sequences are obtained? J. Appl. Probab. 19 (1982) $518-531$.

[5] P. Cénac, Test on the structure of biological sequences via chaos game representation. Stat. Appl. Genet. Mol. Biol. 4 (2005) 36 (electronic).

[6] P. Cénac, G. Fayolle and J.M. Lasgouttes, Dynamical systems in the analysis of biological sequences. Technical Report 5351, INRIA (2004).

[7] M. Drmota, The variance of the height of digital search trees. Acta Informatica 38 (2002) 261-276.

[8] M. Duflo, Random Iterative Models. Springer (1997).

[9] P. Erdős and P. Révész, On the length of the longest head run, in Topics in Information Theory 16 (1975) 219-228, I. Csizàr and P. Elias, Eds. North-Holland, Amsterdam Colloq. Math. Soc. Jànos Bolyai.

[10] P. Erdős and P. Révész, On the length of the longest head-run. In Topics in information theory (Second Colloq., Keszthely, 1975). Colloq. Math. Soc. János Bolyai 16 (1977) 219-228.

[11] J. Fayolle, Compression de données sans perte et combinatoire analytique. Thèse de l'université Paris VI (2006), available at http://www.lri.fr/ fayolle/these.pdf.

[12] J.C. Fu, Bounds for reliability of large consecutive-k-out-of-n:f system. IEEE Trans. Reliability 35 (1986) $316-319$.

[13] J.C. Fu and M.V. Koutras, Distribution theory of runs: a markov chain approach. J. Amer. Statist. Soc. 89 (1994) $1050-1058$.

[14] H. Gerber and S. Li, The occurence of sequence patterns in repeated experiments and hitting times in a markov chain. Stochastic Process. Appl. 11 (1981) 101-108.

[15] N. Goldman, Nucleotide, dinucleotide and trinucleotide frequencies explain patterns observed in chaos game representations of DNA sequences. Nucleic Acids Res. 21 (1993) 2487-2491.

[16] L. Gordon, M.F. Schilling and M.S. Waterman, An extreme value theory for long head runs. Probab. Theory Related Fields 72 (1986) 279-287.

[17] H.J. Jeffrey, Chaos Game Representation of gene structure. Nucleic Acid. Res. 18 (1990) 2163-2170.

[18] M.V. Koutras, Waiting times and number of appearances of events in a sequence of discrete random variables, in Advances in combinatorial methods and applications to probability and statistics, Stat. Ind. Technol., Birkhäuser Boston, Boston, MA (1997) 363-384.

[19] Shuo-Yen Robert Li, A martingale approach to the study of occurrence of sequence patterns in repeated experiments. Ann. Probab. 8 (1980) 1171-1176.

[20] H.M. Mahmoud, Evolution of random search trees. Wiley-Interscience Series in Discrete Mathematics and Optimization. John Wiley \& Sons Inc., New York (1992).

[21] W. Penney, Problem: Penney-ante. J. Recreational Math. 2 (1969) 241.

[22] V. Petrov, On the probabilities of large deviations for sums of independent random variables. Theory Prob. Appl. (1965) $287-298$.

[23] B. Pittel, Asymptotic growth of a class of random trees. Annals Probab. 13 (1985) 414-427. 
[24] V. Pozdnyakov, J. Glaz, M. Kulldorff and J.M. Steele, A martingale approach to scan statistics. Ann. Inst. Statist. Math. 57 (2005) 21-37.

[25] M. Régnier, A unified approach to word occurence probabilities. Discrete Appl. Math. 104 (2000) 259-280.

[26] G. Reinert, S. Schbath and M.S. Waterman, Probabilistic and statistical properties of words: An overview. J. Comput. Biology 7 (2000) $1-46$.

[27] S. Robin and J.J. Daudin, Exact distribution of word occurences in a random sequence of letters. J. Appl. Prob. 36 (1999) 179-193.

[28] A. Roy, C. Raychaudhury and A. Nandy, Novel techniques of graphical representation and analysis of DNA sequences - A review. J. Biosci. 23 (1998) 55-71.

[29] S.S. Samarova, On the length of the longest head-run for a markov chain with two states. Theory Probab. Appl. 26 (1981) 498-509.

[30] V. Stefanov and A.G. Pakes, Explicit distributional results in pattern formation. Ann. Appl. Probab. 7 (1997) 666-678.

[31] W. Szpankowski, Average Case Analysis of Algorithms on Sequences. John Wiley \& Sons, New York (2001).

[32] D. Williams, Probability with martingales. Cambridge Mathematical Textbooks. Cambridge University Press, Cambridge (1991). 